\title{
The population biology and epidemiology of schistosome and geohelminth infections among schoolchildren in Tanzania
}

\author{
M. Booth ${ }^{\star}{ }^{\star}$, C. Mayombana ${ }^{2}$ and P. Kilima ${ }^{3} \quad{ }^{1}$ Department of Public Health and Epidemiology, Swiss Tropical Insti- \\ tute, Socinstrasse 57, CH 4051 Basel, Switzerland; 2 Ifakara Health Research and Development Centre, P.O. Box 53, Ifakara, \\ Tanzania; 3 Ministry of Health, Dar es Salaam, Tanzania
}

\begin{abstract}
A study of helminth infections was undertaken among 3244 schoolchildren from 28 schools in Morogoro Rural District, Tanzania. Schistosoma haematobium was the most common infection, followed by hookworms, Ascaris lumbricoides, S. mansoni, and Trichuris trichiura. Infection prevalence of each species varied among schools and age groups, but not between sexes. There was no relationship between the prevalences of different infections among schools, except for a strong negative correlation between the prevalence of hookworm and $S$. mansoni infections. Within each age group, there was little excess overlap in the distribution of each infection; thus the number of multiple infections was low whereas the number of individuals harbouring at least one infection was relatively high. More children than expected carried infections of $A$. lumbricoides and $S$. mansoni, and the clustering effect increased with age. Only 2 schools had high overall infection prevalences of both geohelminths and schistosomes. Logistic regression analysis of morbidity and parasitological data indicated that individuals with multiple species infections were not at increased risk of morbidity (on a multiplicative scale) compared to individuals with single species infections. This was attributed in part to the low egg counts observed for each parasite species. The results implied little interaction between schistosome and geohelminth infections in the region, both in parasitological terms and in the context of their combined effects on health. Implications for the feasibility and benefits of combined control of geohelminths and schistosomes are discussed.
\end{abstract}

Keywords: geohelminthiasis, schistosomiasis, Ascaris lumbricoides, Trichuris trichiura, hookworms, Schistosoma haematobium, Schistosoma mansoni, prevalence, multiple infections, morbidity, schoolchildren, Tanzania

\section{Introduction}

Ascaris lumbricoides, Trichuris trichiura and hookworm infections are endemic in many Tanzanian communitics, although often only one species is highly prevalent (BOOTH \& BUNDY, 1992). Schistosoma haematobium and $S$. mansoni infections are also endemic (DouMENGE et al., 1987). Both geohelminth and schistosome infections are serious public health problems, and are generally targets of separate control efforts. However, since the 2 types of infection share some factors for transmission, it may be feasible and cost-effective to combine their control if certain criteria are fulfilled.

One important factor supporting combined control is that only 2 drugs are required to treat several species of helminth. Most importantly, though, the geographical distribution of the less common schistosome infections should be contained within the distribution of geohelminth infections (BUNDY et al., 1991). The cost of combined control would obviously be lower if schistosome and geohelminth infections were highly prevalent in the same communities.

In communities where both types of infection are endemic, the benefits of combined intervention will depend partly on the extent and nature of associations among species. For example, the total number of infected individuals treated will depend on the level of infection clustering. ALBONICO et al. (1997) did not find any clustering on Pemba Island, Tanzania; neither did CHUNGE et al. (1995) working in Machakos District, Kenya. DE CleRQ et al. (1995) found fewer infections involving hookworms and schistosomes than expected in Mali, but attributed this to regional differences in transmission factors and climate.

The henefits of intervention can also be measured in terms of alleviating morbidity related to infection. A variety of methods has been applied to estimate the morbidity burden of individual species at the community level (LWAMBO et al., 1992; BOOTH et al., 1996), but it is not clear whether the pathogenic effects of one species may be exacerbated or reduced by the presence of another species in the same host. Since antigenic crossreactivity exists among several helminth species (COR-

*Author for correspondence: phone +41612848427 , fax +41 6712717951 , c-mail booth@ubaclu.unibas.ch
REA-OlIVEIRA et al., 1988; PRITCHARD et al., 1991), there is a potential for this type of interaction, particularly if host immune responses to infection affect pathogenesis.

In this paper, we address the issues of clustering and combined impact on health, by describing the population biology and epidemiology of schistosome and gcohelminth species among children in Morogoro Rural District in Tanzania. Geographical correlations among the prevalences of each infection were assessed, and we examined whether infections with different species were clustered in any one segment of the sampled children at the time of the survey. We also assessed whether individuals with multiple species infections were at higher risk of morbidity than individuals with single species infections. The results are used to comment on the feasibility and potential benefits of combined control of schistosomes and geohelminth infections.

\section{Materials and Methods}

Data collection

Data were collected from several independent surveys of children aged between 7 and 17 years in schools in Morogoro Rural District, Tanzania between October 1992 and June 1993. Details of the district and data collection methods have been given by BOOTH et al. (1998b). Briefly, $A$. lumbricoides, $T$. trichiura and $S$. manson infections were diagnosed by single stool examinations using the Kato-Katz method (KATZ et al., 1972), whereas hookworm infections were detected by the saline flotation method (YANG \& SCHOLTEN, 1977). $S$. haematobium infection was detected by urine filtration.

Questionnaire morbidity surveys were undertaken at the same time as stool collection (BOOTH et al., 1988). Case definitions for the present analysis followed the same scheme as previously described: cases of bloody stool, bloody urine, diarrhoea, and abdominal pain were defined as individuals experiencing the particular symptom during the 2 months before the survey. Liver en largement cases were defined as individuals with a liver extending $2 \mathrm{~cm}$ or more below the right costal margin in the mid-clavicular line. Spleen enlargement cases were defined as individuals with spleens larger than grade 2 on the scale of HACKETT (1944). 


\section{Data analysis}

Prevalences of single and multiple species infections involving $A$. lumbricoides, $T$. trichiura, hookworms, $S$. haematobium and $S$. mansoni were estimated by school and age. Overall infection prevalences of geohelminths and schistosomes were estimated from the number of individuals with at least one species of geohelminth or schistosome infection, respectively.

\section{Infection clustering}

Log linear analysis was used to test whether certain combinations of species infected the same host more often than expected by chance. First, observed numbers of multiplc species infections were compared against figures calculated on the assumption of no excessive clustering using a simple probabilistic model (BOOTH \& BUNDY, 1995). Using a step-wise proccdure, interaction terms between different species, age and sex were then added to the models. Interactions that improved the fit of the model to the data were recorded, and interpreted in terms of excessive infection clustering in a defined population stratum.

\section{Prevalence relationships}

The infection prevalences of each combination of 2 species were compared among the 28 schools. Kendall's rank correlation analysis (KENDALL, 1938) was used to test for the significance of the correlations. The overall infection prevalences of schistosomes and geohelminths in each school were compared using the same procedure.

\section{Egg count associations}

Correlations between egg counts of different parasitic species in hosts with multiple species infections were examined by the rank correlation method of KENDALL (1938). We first considered the complete data set, and then focused on each age class and sex.

Within each age group, the effect of concurrent infections on variation in the egg counts of $A$. lumbricoides or schistosomes was examined with Kruskall-Wallis nonparametric ANOVA (ZAR, 1996). This allowed us to test whether individuals with multiple species infections had higher egg counts of each infection than individuals infected by only one species (BOOTH et al., 1998a). T. trichiura infections were too rare for these analyses, and hookworm eggs were not counted, so could not be evaluated. It was also not possible to accommodate variation in egg counts related to the effect of sampling in 28 different schools due to limitations in the analytical technique.

\section{Effect of multiple species infections on health}

Logistic regression analysis was used to determine whether individuals with multiple species infections were at higher risk of morbidity than individuals with single species infections. As in our previous analysis of the same data (BOOTH et al., 1998b), we included 'school' as a random effect to accommodate variation in the prevalence of each morbidity indicator among the schools. Models consisted of a sign or symptom as the outcome, and explanatory terms for age, sex, and the 2 parasite species of interest. Each model also contained an interaction term between the 2 species. This allowed us to estimate the odds of morbidity in individuals with both infections compared with those in individuals infected by only one species.

\section{Results}

Multiple species infections by age

A total of 3244 children from 28 schools was examined for each geohelminth and schistosome species. Age-prevalence charts for multiple species infections are shown in Fig. 1. The proportion of sampled children with at least one type of schistosome infection remained above $40 \%$ at all ages between 9 and 17 years inclusive,
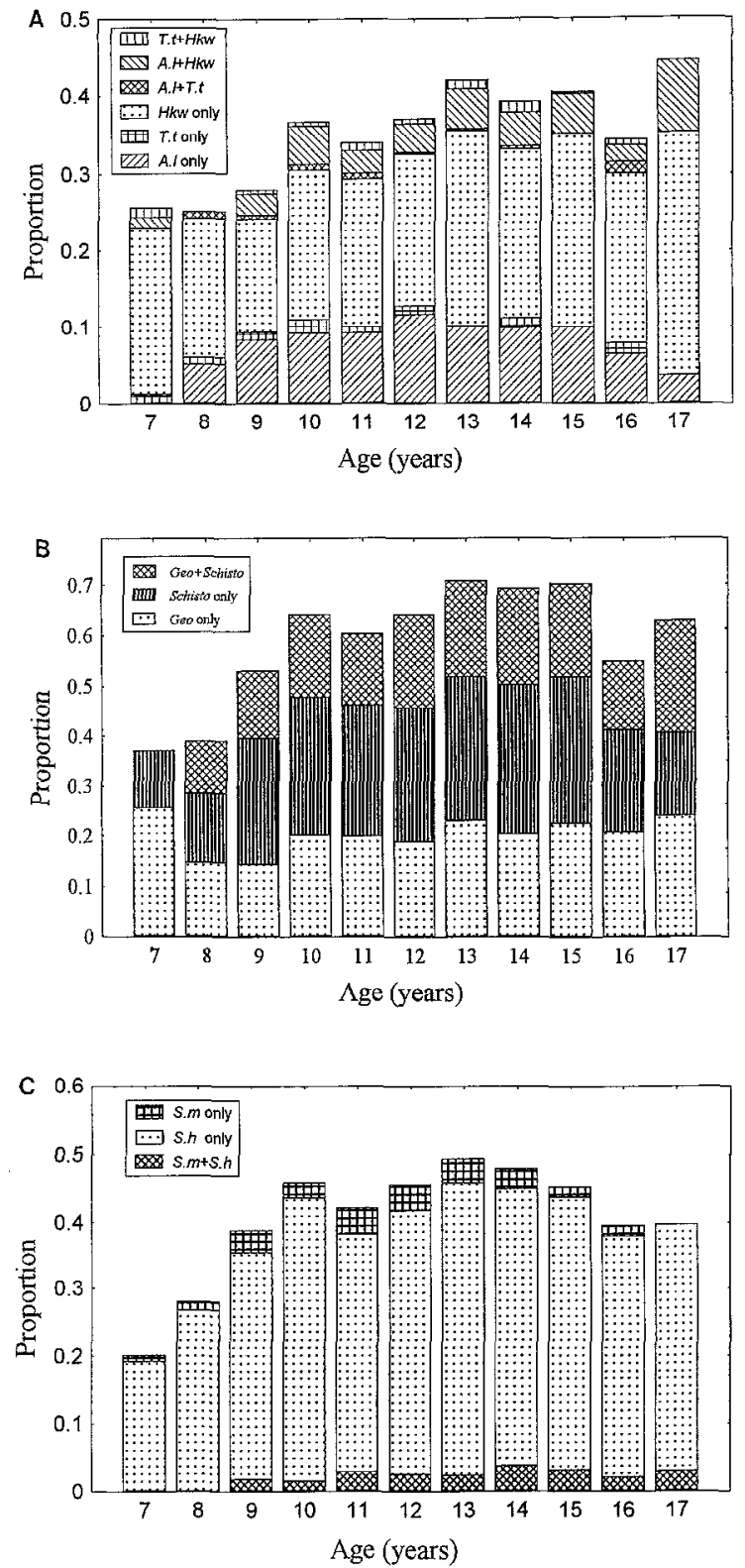

Fig. 1. Relationship between age and prevalence of multiple helminth infections, expressed as stacked bar charts for (A) geohelminths, (B) schistosomes and (C) both types of infection. The segments of each bar represent the proportion of that age group with the combination of infections, as indicated in the legend. The height of each bar represents the total proportion of that age group that was infected. (A.l. $=$ Ascaris humbricoides, Geo=any geohelminth infection, Hkw=hookworms, Schisto $=$ any schistosome infection, $S . h .=S c h i s t o s o m a ~ h a e m a t o b i u m$, S.m. $=$ S. mansoni, T.t. $=$ Trichuris trichiura. )

with a peak of $49 \%$ in the 13 years old children. Similarly, the proportion of the sampled children with at least one helminth infection remained above $30 \%$ between the ages of 10 and 17 years inclusive. Very few children carried both Schistosoma species due to the low prevalence of $S$. mansoni infection, and only 4 children were infected by all 3 geohelminths. The majority of the infected children in each age class carried either geohelminth or schistosome infections, rather than multiple species infections involving a combination of nematode and trematode species (Fig. I, C).

\section{Multiple species infections within schools}

The prevalences of multiple species geohelminth and schistosome infections in each school, ordered by in- 

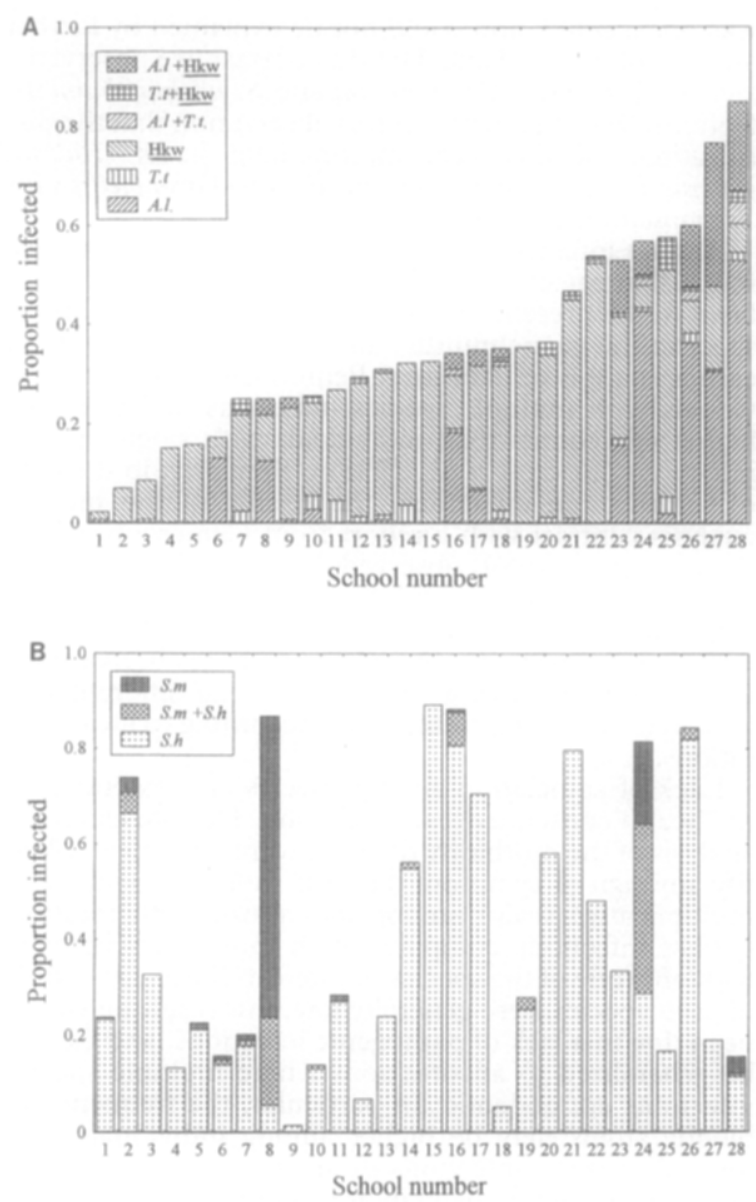

Fig. 2. Proportion of children in each school infected by each combination of (A) geohelminth infections and (B) schistosome infections. The height of each stacked column indicates the overall infection prevalence of each type of infection. Schools are arbitrarily numbered, and ordered according to the overall geohelminth infection prevalence. Infections with all 3 geohelminths were too rare to be displayed. (Abbreviations are as in Fig. 1).
Prevalence relationships

Among the 10 prevalence relationships tested among schools, only one result emerged as significant-the prevalence of hookworm infection was strongly negacively correlated with the prevalence of $S$. mansoni infection (Table 1). Most of the non-significant relationships were positive in nature. Also among schools, there was no significant correlation between the overall infection prevalences of geohelminths and schistosomes (Kendall's $\tau=0 \cdot 109, P=0 \cdot 4173$ )

Infection clustering by age and sex

Log linear analysis revealed that there were more infections involving $A$. lumbricoides and $T$. trichiura than would be expected by chance. The degree of clustering was not affected by age or sex (Table 1). A highly significant intcraction was also found between $A$. lumbricoides and $S$. mansoni, which was affected by age (Table 1 ). This age effect is depicted in Fig. 3, which shows that the observed proportion of each age group with both infections continued to rise with age, whereas the expected proportion reached a peak in the middle age classes. Overall, there were fewer infections involving $S$. manso$n i$ and hookworms than expected by chance; this was at tributed to the negative correlation between infection prevalences of these 2 infections (Table 1).

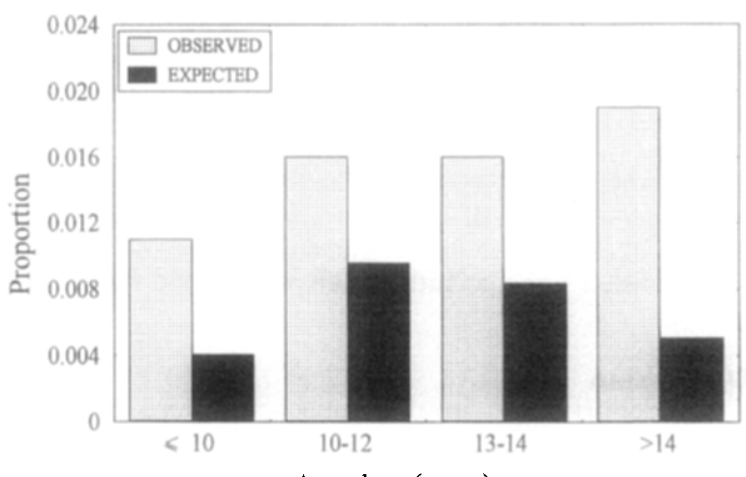

Age class (years)

Fig. 3. Relationship between observed and expected proportion of each age class infected with both $A$. lumbricoides and $S$. monsoni. Expected values were calculated assuming that each infection was transmitted independently.

Table 1. Log linear analysis of whether certain combinations of helminth species occurred more often than expected in schoolchildren and Kendall's rank correlation analysis of prevalences among 28 schools

\begin{tabular}{|c|c|c|c|c|}
\hline \multirow[b]{3}{*}{ Interaction } & \multicolumn{4}{|c|}{ Analysis } \\
\hline & \multicolumn{2}{|c|}{ Log linear } & \multicolumn{2}{|c|}{ Kendall's } \\
\hline & $\chi^{2}$ & $P$ & $\tau$ & $P$ \\
\hline A. lumbricoides + T. trichiura & $8 \cdot 30$ & $0 \cdot 0040^{\mathrm{a}}$ & $0 \cdot 23$ & 0.0830 \\
\hline A. lumbricoides + S. mansoni & $51 \cdot 10$ & $<0 \cdot 0001^{b}$ & $0 \cdot 15$ & $0 \cdot 2547$ \\
\hline A. lumbricoides + S. haematobium & 0.05 & 0.8215 & -0.07 & 0.5836 \\
\hline T. trichiura + S. haematobium & $2 \cdot 05$ & $0 \cdot 1527$ & $0 \cdot 00$ & 1.0000 \\
\hline T. trichiura $+S$. mansoni & $0 \cdot 40$ & 0.5297 & $0 \cdot 05$ & $0 \cdot 7016$ \\
\hline S. haematobium + S. mansoni & $2 \cdot 45$ & 0.1172 & 0.04 & 0.7852 \\
\hline A. lumbricoides + hookworms & $2 \cdot 2$ & $0 \cdot 1388$ & -0.01 & 0.9328 \\
\hline T. trichiura + hookworms & $10 \cdot 0$ & $0 \cdot 0016^{a, c}$ & $0 \cdot 21$ & $0 \cdot 1231$ \\
\hline S. haematobium + hookworms & $1 \cdot 6$ & $0 \cdot 2014$ & 0.01 & 0.9370 \\
\hline S. mansoni + hookworms & $13 \cdot 2$ & $0.0003^{a, c}$ & -0.47 & $0 \cdot 0004$ \\
\hline
\end{tabular}

aNo significant age or sex effect.

bSignificant age effect $\left(\chi^{2}=8 \cdot 06, P=0 \cdot 0449\right)$.

cFewer infections than expected.

creasing overall prevalence of geohelminth infection, are shown in Fig. 2. In 10 schools the overall infection prevalence of schistosomes was greater than $50 \%$, and in 7 schools the overall infection prevalence of geohelminths was higher than this value. However, there were only 2 schools in which the overall prevalence of both types of infection was above $50 \%$.

\section{Egg count associations}

Six relationships between the egg counts of different species were examined (Table 2). The only significant result was an apparent negative correlation between $S$. mansoni and $S$. haematobium infection intensities. However, when an age-stratified analysis was performed, the correlation remained significant only among infected 
Table 2. Kendall's rank correlation analysis of egg counts of different helminth species in schoolchildren infected by both species

\begin{tabular}{lccc}
\hline & $\begin{array}{c}\text { No. of } \\
\text { children } \\
\text { infected }\end{array}$ & $\begin{array}{c}\tau \\
\text { Combination }\end{array}$ & $P$ \\
\hline S. haematobium + S. mansoni & 109 & -0.14 & 0.0293 \\
S. haematobium + A. lumbricoides & 231 & 0.01 & 0.7703 \\
S. haematobium + T. trichiura & 39 & 0.06 & 0.5766 \\
S. mansoni + A. lumbricoides & 68 & 0.10 & 0.2256 \\
S. mansoni + T. trichiura & 5 & 0.67 & 0.1025 \\
A. lumbricoides + T. trichiura & 22 & -0.33 & 0.8314 \\
\hline
\end{tabular}

individuals in the $10-12$ years age class $(n=36$, $\tau=-0.275, P=0.0186)$. This was attributed to the concurrent decrease in mean $S$. haematobium infection intensity and increase in mean $S$. mansoni infection intensity in this age class (BOOTII et al., 1998b). All oth er comparisons within age groups or sex were non-significant. Further, there was no evidence that egg counts of A. lumbricoides, S. haematobium or S. mansoni infections varied significantly depending on the number or type of concurrent infections that were present in a host.

\section{Effects of multiple infections on health}

The risk of morbidity for each sign and symptom was compared among individuals with single and multiple species infections, for each combination of 2 infections when there were enough infected cases. This process yielded a total of 58 adjusted odds ratios, none of which was significantly greater than one. These results mean that individuals with multiple species infections were equally likely to have experienced defined symptoms, or have defined signs, as individuals with single species infections.

\section{Discussion}

Few reports have simultaneously considered the population biology of schistosomes and geohelminth infections, despite the fact that multiple species infections are often endemic in the same community. This may be because several important ecological factors differ between the 2 species, in particular their modes of transmission are quite distinct, and thus research efforts have been largely separated. None the less, some risk factors for transmission are shared, and in theory at least this may cause the 2 types of infection to be associated by concentration in the same small geographical area, and/ or clustering within certain population strata. The present study assessed this issue in detail, and is particularly relevant in the context of assessing the overall public health importance of multiple helminth infections in endemic areas and the general prospects for the combined control of schistosome and geohelminth infections.

In terms of geographical distribution, the 2 types of infections were independent, apart from the strong negative correlation between the prevalence of hookworm and $S$. mansoni infections. In Morogoro Region, factors for successful transmission of $S$. mansoni and hookworms must therefore be exclusive to some degree. Most probably, factors related to sampling schools at different altitudes are important, since independent studies have demonstrated that altitude affects directly or indirectly the distribution of both types of infection (NORDBECK et al., 1982; BIRRIE et al., 1994; APPLETON \& GOUWS, 1996).

Within communities, there was no excessive clustering of geohelminth infections, apart from $A$. lumbricoides and T. trichiura. This observation is consistent with those from other areas, and is probably due to similarities in the transmission mechanisms of these 2 species (BOOTH \& BUNDY, 1995). The increased clustering of $A$. lumbricoides and $S$. mansoni infections with host age was unexpected, and could not be explained by further analysis of the available data. In contrast, we observed a lack of clustering of $S$. mansoni and $S$. haematobium infections. We can infer from this observation that the distributions of the snail intermediate hosts (Bulinus globosus for S. haematobium and Biomphalaria pfeifferi for $S$. mansoni) are independent.

Among infected individuals within communities, wc found that egg counts of individual species were not related to the presence of concurrent infections. With respect to the gcohclminths, this result contrasts with an earlier analysis of data from Pemba Island, where individuals with multiple species infections had generally higher egg counts than individuals with single species infections (BOOTH et al., 1998a). Similarly, in the case of schistosome infections, the results of the present analysis contrast with a report from Cameroon, where ROBERT et al. (1989) observed that individuals infected with both $S$. mansoni and $S$. haematobium had higher egg counts of both species than individuals infected with only one parasite species. From these observations, we can conclude that associations among different species, in terms of egg counts, vary according to local conditions.

Lack of association among species also extended to the health consequences of infection. Despite extensive analysis of the morbidity data, we were not able to identify any sign or symptom that was more frequent (on a multiplicative scale) among individuals with multiple species infections compared with individuals infected with only one of the species of interest. Since egg counts of each species were generally low, this result may have been due to a lack of pathogenic infections, and cannot be generalized to all situations where multiple species infections are endemic. In particular, the frequency of bloody stools may be higher among individuals with multiple species infections in areas where $T$. trichiura, hookworms and $S$. mansoni infections are more prevalent, since this symptom can be caused by each of these infections (SLEISENGER \& FORDTRAN, 1989).

We can conclude from the above observations that schistosome and geohelminth infections are effectively independent in terms of geographical distribution, and pathogenic effects, in Morogoro. However, this does not mean that control efforts should necessarily remain independent, in Morogoro or elsewhere with a similar situation. Although the criteria for cost-effective combined control, set by BUNDY et al. (1991) and WARREN et al. (1993), may not be fulfilled, it may still be feasible to plan the control of each infection within a single intervention. Since school-aged children are usually at highest risk of schistosome and geohelminth infection, control efforts could be focused on this age group. Further identification of which schools require which drugs could be undertaken with rapid assessment techniques, at least for the schistosomes (LENGELER et al., 1991; BOOTH et al., 1998b), thus reducing screening costs.

We can also use the results of the present study to comment on the potential benefits of combined intervention in an area with a similar situation to Morogoro-namely, where the infection prevalence of most species is low, there is little infection clustering, and there is no inter-species interaction. In these circumstances, most infected individuals will have a low multiplicity of infection, and the severity of morbidity in a host will be a linear function of the number of species harboured. This effectively means that the total morbidity burden attributable to helminth infections will be distributed widely among the infected individuals. Similarly, the health benefits of a combined intervention would be shared. Since the drugs of choice against schistosome and geohelminth infections have a broad spectrum of activity (DE SILVA et al., 1997), there may be additional benefits to community health even if some individuals have no schistosome or geohelminth infection at the time of intervention. 
It is not possible within the scope of the present analysis to present a definitive statement on the feasibility, cost-effectiveness and benefits of combined control. None the less, the conclusions are useful in the context of planning such an operation. It is also important to note that different regions have different transmission levels of individual species, which will affect the amount of infection clustering within and among communities. In particular, the highly focal distribution of schistosome infections may affect the level of infection clustering with geohelminth species over a small geographical area. Morbidity patterns will vary according to local conditions and the number of endemic parasite species, as will rates of re-infection. All these factors will contribute to the feasibility and benefits of combined control in a particular location, and they imply that control strategics should be planncd and implemented at a local rather than national level.

\section{Acknowledgements}

We thank the project team, authorities, teachers and students in Morogoro, and Professor W. Kilama, former director of the National Institute of Medical Research, Tanzania. The project received financial support from the UNDP/World Bank/WHO Special Programme for Research and Training in Tropical Diseases (TDR project ID 920129). M.B. was supported by a Wellcome Trust Travelling Research Fellowship.

\section{References}

Albonico, M., Chwaya, H. M., Montresor, A., Stoltzfus, R. J., Tielsch, J. M., Alawi, K. S. \& Savioli, L. (1997). Parasitic infections in Pemba Island schoolchildren. East African Medical fournal, 74, 294-298.

Appleton, C. C. \& Gouws, E. (1996). The distribution of common intestinal nematodes along an altitudinal transect in KwaZulu-Natal, South Africa. Annals of Tropical Medicine and Parasitology, 90, 181-188.

Birrie, H., Erko, B. \& Tedla, S. (1994). Intestinal helminthic infections in the southern rift valley of Ethiopia with special reference to schistosomiasis. East African Medical Fournal, $71,447-452$.

Booth, M. \& Bundy, D. A. P. (1992). Comparative prevalences of Ascaris lumbricoides, Trichuris trichizera and hookworm infections and the prospects for combined control. Parasitology, 105, 151-157.

Booth, M. \& Bundy, D. A. P. (1995). Estimating the number of multiple-species geohelminth infections in human communities. Parasitology, 111, 645-653.

Booth, M., Guyatt, H. L., Li, Y. \& Tanner, M. (1996). The morbidity attributable to Schistosoma japonicum infection in 3 villages in Dongting Lake region. Hunan Province, China. Tropical Medicine and International Health, 1, 646-654.

Booth, M., Bundy, D. A. P., Albonico, M., Chwaya, H. M., Alawi, K. S. \& Savioli, L. (1998a). Associations among multiple geohelminth species infections in schoolchildren from Pemba Island. Parasitology, 116, 85-93.

Booth, M., Mayombana, C., Machibya, H., Masanja, H., Odermatt, P., Utzinger, J. \& Kilima, P. (1998b). The use of morbidity questionnaires to identify communities with high prevalences of schistosome or geohelminth infections in Tanzania. Transactions of the Royal Society of Tropical Medicine and Hygiene, 92, 484-490.

Bundy, D. A. P., Chandiwana, S. K., Homeida, M. M. A. Yoon, S. \& Mott, K. E. (1991). The epidemiological implications of a multiple-infection approach to the control of human helminth infections. Transactions of the Royal Society of Tropical Medicine and Hygiene, 85, 274-276.

Chunge, R. N., Karumba, N., Ouma, J. H., Thiongo, F. W., Sturrock, R. F. \& Butterworth, A. E .(1995). Polyparasitism in two rural communities with endemic Schistosoma mansoni infection in Machakos District, Kenya. Journal of Tropical Medicine and Hygiene, 98, 440-444.

Correa-Oliveira, R., Dusse, L. M., Viana, I. R., Colley, D. G., Santos Carvalho, O. \& Gazzinelli, G. (1988). Human antibody responses against schistosomal antigens. I. Antibodies from patients with Ancylostoma, Ascaris lumbricoides or Schistosoma mansoni infections react with schistosome antigen. American fournal of Tropical Medicine and Hygiene, 38, 348-355.

De Clerq, D., Sacko, M., Behnke, J. M. \& Vercruysse, J. (1995). Schistosoma and geohelminth infections in Mali, West Africa, Annales de la Société Belge de Médecine Tropicale, $75,191-199$.

De Silva, N., Guyatt, H. \& Bundy, D. (1997). Anthelmintics. A comparative review of their clinical pharmacology. Drugs, 53, 769-788.

Doumenge, J. P., Mott, K. E., Cheung, C., Villenave, D. \& Chapuis, O. (1987). Atlas of the Global Distribution of Schistosomiasis. Geneva: World Health Organization.

Hackett, L. W. (1944). Spleen measurement in malaria. fournal of the National Malaria Society, 3, 121-123.

Katz, N., Chaves, A. \& Pellegrino, J. (1972). A simple device for quantitative stool thick smear technique in schistosomiasis mansoni. Revista do Instituto de Medicina Tropical de São Paulo, 14, 397-400.

Kendall, M. G. (1938). A new measure of rank correlation. Biometrika, 30, 81-93.

Lengeler, C., Kilima, P., Mshinda, H., Morona, D., Hatz, C. \& Tanner, M. (1991). Rapid, low-cost, two step method to screen for urinary schistosomiasis at the district level: the Kilosa experience. Bulletin of the World Health Organization, 69, $179-189$

Lwambo, N. J. S., Bundy, D. A. P. \& Medley, G. F. H. (1992). A new approach to morbidity risk assessment in hookworm endemic communities. Epidemiology and Infection, 108, 469-481.

Nordbeck, H. J., Ouma, J. H. \& Slooff, R. (1982). Machakos project studies. Agents affecting health of mother and child in a rural area of Kenya. XXII. Schistosomiasis transmission in relation to some socio-economic and other environmental factors. Tropical and Geographical Medicine, 34, 193-203.

Pritchard, D. I., Quinnell, R. J., McKean, P. G., Walsh, L., Leggett, K. V., Slater, A. F. G., Raiko, A., Dale, D. D. S. \& Keymer, A. E. (1991). Antigenic cross-reactivity between Necator americanus and Ascaris lumbricoides in a community in Papua New Guinea infected predominantly with hookworm. Transactions of the Royal Society of Tropical Medicine and Hygiene, 85, 511-514.

Robert, C. F., Bouvier, S. \& Rougemont, A. (1989). Epidemiology of schistosomiasis in the riverine population of Lagdo Lake, northern Cameroon: mixed infections and ethnic factors. Tropical Medicine and Parasitology, 40, 153-158.

Sleisenger, M. H. \& Fordtran, J. S. (1989). Gastrointestinal Disease, volume 1: Pathophysiology, Diagnosis, Managernent. Philadelphia:W. B. Saunders

Warren, K. S., Bundy, D. A. P., Anderson, R. M., Davis, A. R., Henderson, D. A., Jamison, D. T., Prescott, N. \& Senft, A. (1993). Helminthic infections. In: Disease Control Priorities in Developing Countries, Jamison, D. T., Mosely, W. H., Measham, A. R. \& Bobadilla, J. L. (editors). Oxford: Oxford University Press, pp. 131-160.

Yang, J. \& Scholten, T. A. (1977). A fixative for intestinal parasites permitting the use of concentration and permanent staining procedures. American fournal of Clinical Pathology, $67,300-304$.

Zar, J. H. (1996). Biostatistical Analysis. New Jersey: Prentice Hall.

Received 23 February 1998; revised 18 May 1998; accepted for publication 19 May 1998 